\title{
Effect of an orientation-dependent non-linear grain fluidity on bulk directional enhancement factors
}

\section{Letter}

Cite this article: Rathmann NM, Hvidberg CS, Grinsted A, Lilien DA, Dahl-Jensen D (2021). Effect of an orientation-dependent non-linear grain fluidity on bulk directional enhancement factors. Journal of Glaciology 67(263), 569-575. https://doi.org/10.1017/jog.2020.117

Received: 9 September 2020

Revised: 16 December 2020

Accepted: 17 December 2020

First published online: 28 January 2021

\section{Keywords:}

Anisotropic ice; ice rheology; ice-sheet modelling

Author for correspondence:

Nicholas M. Rathmann,

E-mail: rathmann@nbi.ku.dk
Nicholas M. Rathmann (D), Christine S. Hvidberg, Aslak Grinsted, David

A. Lilien (I) and Dorthe Dahl-Jensen (i)

Niels Bohr Institute, University of Copenhagen, Copenhagen, Denmark

\section{Abstract}

Bulk directional enhancement factors are determined for axisymmetric (girdle and single-maximum) orientation fabrics using a transversely isotropic grain rheology with an orientationdependent non-linear grain fluidity. Compared to grain fluidities that are simplified as orientation independent, we find that bulk strain-rate enhancements for intermediate-to-strong axisymmetric fabrics can be up to a factor of ten larger, assuming stress homogenization over the polycrystal scale. Our work thus extends previous results based on simple basal slip (Schmid) grain rheologies to the transversely isotropic rheology, which has implications for large-scale anisotropic ice-flow modelling that relies on a transversely isotropic grain rheology. In order to derive bulk enhancement factors for arbitrary evolving fabrics, we expand the caxis distribution in terms of a spherical harmonic series, which allows the rheology-required structure tensors through order eight to easily be calculated and provides an alternative to current structure-tensor-based modelling.

\section{Introduction}

The viscosity of polycrystalline ice depends on physical properties such as the orientation distribution of individual grains (orientation fabric) (Shoji and Langway, 1985, 1988), grain sizes (Goldsby and Kohlstedt, 1997; Kuiper and others, 2020a, 2020b; Fan and others, 2020), and hardening effects due to crystal lattice defects (Faria and others (2014) and references therein). The orientation fabric is particularly important because ice monocrystals deform by dislocation slip (shear) along crystallographic planes (Fig. 1 left), where basal plane shear (planes with normal c) is up to 100-1000 times softer than along any other plane (Weertman, 1973; Duval and others, 1983). As a consequence, anisotropic orientation fabrics can introduce local directional hardening of a polycrystal (Thorsteinsson $(2001,2002)$ among others), which in turn can affect the deformation of ice masses at large scales (Thorsteinsson and others, 2003; Gillet-Chaulet and others, 2006; Pettit and others, 2007; Martín and Gudmundsson, 2012; Hruby and others, 2020).

General monocrystal rheologies for ice, such as those proposed by Castelnau and others (2008) and Suquet and others (2012), should be able to account for the variation in shear resistance that occurs between different slip systems, including the observed non-linear power law behaviour (Kamb, 1961; Duval and others, 1983). A popular simplified rheology is the transversely isotropic rheology of Johnson (1977) (see Supplementary A and the notation section):

$$
\begin{aligned}
& \dot{\boldsymbol{\epsilon}}^{\prime}=\eta^{-1}\left(\boldsymbol{\tau}^{\prime}-\frac{E_{c c}^{\prime}-1}{2}\left(\boldsymbol{\tau}^{\prime} \cdot . \mathbf{c c}\right) \mathbf{I}+\frac{3\left(E_{c c}^{\prime}-1\right)-4\left(E_{c a}^{\prime}-1\right)}{2}\left(\boldsymbol{\tau}^{\prime} \cdot . \mathbf{c c}\right) \mathbf{c c}\right. \\
& \left.+\left(E_{c a}^{\prime}-1\right)\left(\boldsymbol{\tau}^{\prime} \cdot \mathbf{c c}+\mathbf{c c} \cdot \boldsymbol{\tau}^{\prime}\right)\right) \\
& \eta^{-1}=A^{\prime}\left(\boldsymbol{\tau}^{\prime} \cdot \boldsymbol{\tau}^{\prime}+\frac{3\left(E_{c c}^{\prime}-1\right)-4\left(E_{c a}^{\prime}-1\right)}{2}\left(\boldsymbol{\tau}^{\prime} \cdot \cdot \mathbf{c c}\right)^{2}+2\left(E_{c a}^{\prime}-1\right) \boldsymbol{\tau}^{\prime 2} \cdot \cdot \mathbf{c c}\right)^{\left(n^{\prime}-1\right) / 2}
\end{aligned}
$$

which approximates monocrystals as axisymmetric with symmetry axis c (Meyssonnier and Philip, 1996). Here $\boldsymbol{\tau}^{\prime}$ and $\dot{\boldsymbol{\epsilon}}^{\prime}$ are the microscopic deviatoric stress and strain-rate tensors, $A^{\prime}$ is an isotropic rate factor, and $n^{\prime}$ is the power-law exponent. The enhancement factors $E_{c c}^{\prime}$ and $E_{c a}^{\prime}$ are the strain-rate enhancements for compression/extension along $\mathbf{c}$ and shear parallel to basal planes (Fig. 1):

$$
\dot{\boldsymbol{\epsilon}}_{c c}^{\prime}=\dot{\boldsymbol{\epsilon}}^{\prime} \cdot \cdot \mathrm{cc}=\eta^{-1} E_{c c}^{\prime} \tau_{c c}^{\prime},
$$






Fig. 1. Left: Monocrystal lattice composed of hexagonal cells. Three crystallographic planes are highlighted in gray, where the c-axis indicates the basal-plane normal direction. Right: Monocrystal modelled as a transversely isotropic material with symmetry axis $\mathbf{c}$ and longitudinal $\left(E_{c c}^{\prime}\right)$ and shear $\left(E_{c a}^{\prime}\right)$ enhancement factors w.r.t. c. The transverse direction, $\mathbf{a}$, lies in the plane of isotropy $(\mathbf{a} \perp \mathbf{c})$.

$$
\dot{\boldsymbol{\epsilon}}_{c a}^{\prime}=\dot{\boldsymbol{\epsilon}}^{\prime} \cdot \cdot \mathbf{c a}=\eta^{-1} E_{c a}^{\prime} \tau_{c a}^{\prime}
$$

where a denotes any direction transverse to $\mathbf{c}$, and the indices $c$ and $a$ indicate the tensorial components in the direction of $\mathbf{c}$ and $\mathbf{a}$, respectively.

By averaging the rheology (1)-(2) over a polycrystal (defined below), it is possible to construct a bulk (polycrystalline) rheology that provides an anisotropic extension to Glen-Nye's isotropic flow law (Svendsen and Hutter, 1996), or to infer bulk directional enhancement factors, the latter a prerequisite for bulk rheologies such as the orthotropic flow law proposed by Gillet-Chaulet and others (2005). Past work has, however, focused on the linear $\left(n^{\prime}=1\right)$ monocrystal rheology (Gillet-Chaulet and others, 2005, 2006; Durand and others, 2007) or neglected the orientationdependent contributions to the non-linear fluidity $\eta^{-1}$ (Pettit and others, 2007; Martín and others, 2009; Ma and others, 2010; Martín and Gudmundsson, 2012; Hruby and others, 2020) by setting

$$
\eta^{-1} \simeq A^{\prime}\left(\boldsymbol{\tau}^{\prime} \cdot . \boldsymbol{\tau}^{\prime}\right)^{\left(n^{\prime}-1\right) / 2}
$$

For strongly developed fabrics, bulk directional enhancement factors calculated using (1) with (5) (Gödert and Hutter, 1998; Gagliardini and Meyssonnier, 1999) can meanwhile lead to smaller enhancements compared to basal slip (Schmid) models that include an orientation-dependent fluidity (Thorsteinsson, 2001). Bulk rheologies that rely on (5) as the grain fluidity might therefore produce different flow regimes compared to (2).

It is not clear over which regimes of orientation fabric strength (5) provides a good approximation to (2) (in the grain-averaged rheology), or what fabric information the grain-averaged rheology requires if (2) is to be used. In this letter, we address these questions by calculating bulk directional enhancement factors for single-maximum and girdle fabrics using both the full (2) and approximate (5) fluidities. In doing so, we find that the full fluidity (2) requires specifying more detailed information about the orientation distribution of grains (higher-order structure tensors), which leads us to propose a new non-parametric spectral fabric model instead of directly modelling the structure tensors.

\section{Notation}

Throughout, primes shall be used to denote rheological parameters, stresses and strain rates of monocrystals, as opposed to non-primed variables used to denote bulk rheological parameters, stresses and strain rates of polycrystals. Boldface symbols denote vectors or tensors, the order of the latter being implicit by the context. Let $\mathbf{a}$ and $\mathbf{b}$ be vectors, and $\mathbf{A}$ and $\mathbf{B}$ be second-order tensors. The following products are then used: $\mathbf{a} \cdot \mathbf{b}=a_{i} b_{i}, \mathbf{a b}=$ $a_{i} b_{j}$ (the outer, dyadic product), $\mathbf{A} \cdot \mathbf{a}=A_{i j} a_{j}, \mathbf{A} \cdot \mathbf{a b}=A_{i j} a_{j} b_{i}$,
$\mathbf{A} \cdot \mathbf{B}=A_{i j} B_{j k}$, and $\mathbf{A} \cdot \mathbf{B}=A_{i j} B_{j i}=\operatorname{tr}(\mathbf{A} \cdot \mathbf{B})$, where summation over repeated indices is implied. The identity matrix is denoted by $\mathbf{I}$, and the superscript $\mathrm{T}$ denotes the matrix transpose.

\section{Averaged rheology}

Following Azuma (1994) (among others), we define the bulk strain rate, $\dot{\boldsymbol{\epsilon}}$, as the average strain rate of grains in a polycrystal. Moreover, we adopt the Sachs hypothesis (Sachs, 1929) that the microscopic stress field, $\boldsymbol{\tau}^{\prime}$, can be assumed homogeneous over the polycrystal and equal to the bulk stress at the polycrystal scale, $\boldsymbol{\tau}$. Taken together, the bulk strain rate is therefore

$$
\dot{\boldsymbol{\epsilon}}=\left\langle\dot{\boldsymbol{\epsilon}}^{\prime}\left(\boldsymbol{\tau}^{\prime}\right)\right\rangle=\left\langle\dot{\boldsymbol{\epsilon}}^{\prime}(\boldsymbol{\tau})\right\rangle
$$

By specifying the individual grain stresses in this way, grain strain compatibility (aligned shared grain boundaries) is not guaranteed, and mechanical interactions between grains are not directly accounted for.

The Sachs hypothesis can broadly be regarded as an endmember case along a spectrum of homogenization schemes. At the opposite end, the Taylor hypothesis (Taylor, 1938) assumes a homogeneous strain rate over the polycrystal. The Taylor hypothesis is, however, not well adapted for strongly anisotropic fabrics: five independent slip systems are required to accommo-

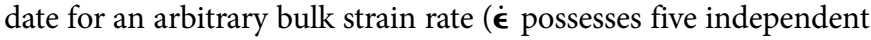
components), but basal planes provide only two (Castelnau and others, 1996). Although the realized stress and strain-rate fields inside polycrystals are somewhere between the two end-member cases, observations and experiments (Azuma and Higashi, 1985; Azuma, 1995) suggest that assuming a homogeneous stress field is a better approximation of the two. For brevity, we refer the reader to Thorsteinsson (2001), Castelnau and others (1996) or Montagnat and others (2014) for details and more sophisticated homogenization schemes.

Given a prescribed stress that all grains are subject to, equation (6) reduces to an average over all grain orientations. Provided with a (unnormalized) grain c-axis distribution $n(\theta, \phi)$ defined over orientation space $S^{2}$, the average strain rate of grains becomes

$$
\left\langle\dot{\boldsymbol{\epsilon}}^{\prime}(\boldsymbol{\tau})\right\rangle_{n(\theta, \phi)}=\frac{1}{N} \int_{S^{2}} \dot{\boldsymbol{\epsilon}}^{\prime}(\boldsymbol{\tau}) n(\theta, \phi) \mathrm{d} \Omega,
$$

where $\mathrm{d} \Omega=\sin \theta \mathrm{d} \theta \mathrm{d} \phi$ is the infinitesimal solid angle, and

$$
N=\int_{S^{2}} n(\theta, \phi) \mathrm{d} \Omega
$$

is the total number of grains. Unless stated otherwise, $\langle\cdot\rangle=\langle\cdot\rangle_{n(\theta, \phi)}$ is hereafter assumed implicit. (6) is

Averaging the rheology (1)-(2) using (7), the bulk strain rate

$$
\begin{aligned}
\left\langle\dot{\boldsymbol{\epsilon}}^{\prime}(\boldsymbol{\tau})\right\rangle=\left\langle\boldsymbol{\eta}^{-1}\right\rangle \boldsymbol{\tau} & -\frac{E_{c c}^{\prime}-1}{2}\left(\boldsymbol{\tau} \cdot\left\langle\left\langle\eta^{-1} \mathbf{c}^{2}\right\rangle\right) \mathbf{I}\right. \\
+ & \frac{3\left(E_{c c}^{\prime}-1\right)-4\left(E_{c a}^{\prime}-1\right)}{2} \boldsymbol{\tau} \cdot \cdot\left\langle\eta^{-1} \mathbf{c}^{4}\right\rangle \\
& \quad+\left(E_{c a}^{\prime}-1\right)\left(\boldsymbol{\tau} \cdot\left\langle\eta^{-1} \mathbf{c}^{2}\right\rangle+\left\langle\eta^{-1} \mathbf{c}^{2}\right\rangle \cdot \boldsymbol{\tau}\right),
\end{aligned}
$$

where $\mathbf{c}^{k}$ is the $k$-th outer (dyadic) product of $\mathbf{c}$ with itself. In the linear case, $n^{\prime}=1$, the averaged terms reduce to $\left\langle\eta^{-1} \mathbf{c}^{k}\right\rangle=\left\langle A^{\prime} \mathbf{c}^{k}\right\rangle=A^{\prime}\left\langle\mathbf{c}^{k}\right\rangle$, and determining $\left\langle\dot{\boldsymbol{\epsilon}}^{\prime}\right\rangle$ therefore requires constructing the second- and fourth-order structure tensors, $\left\langle\mathbf{c}^{2}\right\rangle$ and $\left\langle\mathbf{c}^{4}\right\rangle$ (Advani and Tucker, 1987). Likewise, for the 


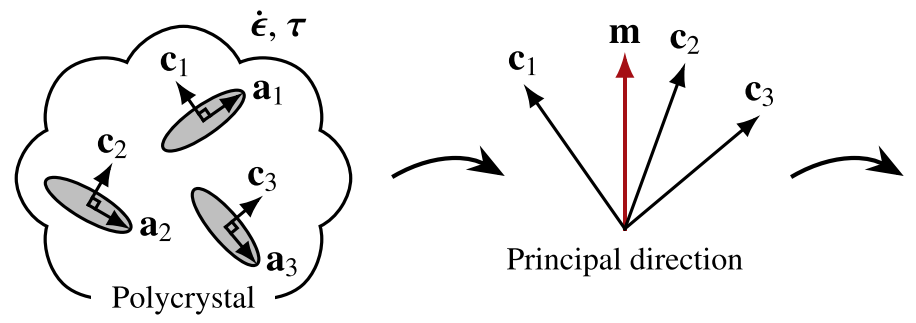

orientation-independent fluidity (5), the averaged terms reduce to $\left\langle\eta^{-1} \mathbf{c}^{k}\right\rangle=A^{\prime}\left(\boldsymbol{\tau}^{\prime} . . \boldsymbol{\tau}^{\prime}\right)^{\left(n^{\prime}-1\right) / 2}\left\langle\mathbf{c}^{k}\right\rangle$, and hence $\left\langle\dot{\boldsymbol{\epsilon}}^{\prime}\right\rangle$ depends on the same structure tensors.

As noted, the rheology of monocrystals has been experimentally found to follow a power law with an exponent of 2 to 3 (Duval and others, 1983). In the non-linear case of $n^{\prime}=3$, the averaged terms in (9) become (for any integer $k$ )

$$
\begin{aligned}
\left\langle\eta^{-1} \mathbf{c}^{k}\right\rangle=A^{\prime} & \left((\boldsymbol{\tau} \cdot \boldsymbol{\tau})\left\langle\mathbf{c}^{k}\right\rangle\right. \\
+ & \frac{3\left(E_{c c}^{\prime}-1\right)-4\left(E_{c a}^{\prime}-1\right)}{2} \boldsymbol{\tau} \cdot \cdot\left\langle\mathbf{c}^{k+4}\right\rangle \cdot \cdot \boldsymbol{\tau} \\
& \left.+2\left(E_{c a}^{\prime}-1\right) \boldsymbol{\tau}^{2} \cdot \cdot\left\langle\mathbf{c}^{k+2}\right\rangle\right),
\end{aligned}
$$

and determining $\left\langle\dot{\boldsymbol{\epsilon}}^{\prime}\right\rangle$ therefore requires constructing the structure tensors $\left\langle\mathbf{c}^{2}\right\rangle,\left\langle\mathbf{c}^{4}\right\rangle,\left\langle\mathbf{c}^{6}\right\rangle$ and $\left\langle\mathbf{c}^{8}\right\rangle$. That is, adopting the orientation-dependent fluidity (2) with $n^{\prime}=3$ over the orientation-independent fluidity (5) requires additionally specifying the higher-order structure tensors $\left\langle\mathbf{c}^{6}\right\rangle$ and $\left\langle\mathbf{c}^{8}\right\rangle$.

We mention in passing that while considering the Taylor hypothesis, too, would provide a more complete treatment of the problem, averaging the inverse rheology, $\left\langle\boldsymbol{\tau}^{\prime}(\dot{\boldsymbol{\epsilon}})\right\rangle$ (see Supplementary A), for $n^{\prime}>1$ involves inverse fractional integrands that are hard to rewrite in terms of even-ordered structure tensors in analogy to (10).

With increasing order, structure tensors quantify the structure of $n(\theta, \phi)$ at an increasingly finer scale on $S^{2}$. It is, however, not clear over which regimes of fabric strength that (5) is a good approximation to (2). In what follows, we address this question by calculating bulk enhancement factors both assuming (5) and while relaxing this assumption.

\section{Bulk directional enhancement factors}

The rheology of an anisotropic polycrystal can be characterized by its bulk enhancement factors for compression/extension and shear w.r.t. the fabric principal directions. Given $\left\langle\dot{\boldsymbol{\epsilon}}^{\prime}(\boldsymbol{\tau})\right\rangle$, Thorsteinsson (2001) suggested defining the bulk enhancement of the $\mathbf{v}-\mathbf{w}$ component of $\dot{\boldsymbol{\epsilon}}$ as ( $\mathbf{v}$ and $\mathbf{w}$ being arbitrary vectors)

$$
E_{v w}(\boldsymbol{\tau})=\frac{\left\langle\dot{\boldsymbol{\epsilon}}^{\prime}(\boldsymbol{\tau})\right\rangle \cdot \cdot \mathbf{v w}}{\left\langle\dot{\boldsymbol{\epsilon}}^{\prime}(\boldsymbol{\tau})\right\rangle_{\text {const. }} \cdot \mathbf{v w}},
$$

where $\left\langle\dot{\boldsymbol{\epsilon}}^{\prime}(\boldsymbol{\tau})\right\rangle_{\text {const. }}$ is the average strain-rate tensor of an isotropic polycrystal $(n(\theta, \phi)=$ const. $)$.

It is important to note that $E_{v w}$ does not depend on $A^{\prime}$ or the stress magnitude because they are orientation independent and therefore cancel by virtue of the division in (11). Likewise, if $\eta^{-1}$ is approximated by (5) (orientation independent), then $E_{v w}$ does not depend on $\eta^{-1}$ either. As a consequence, $E_{v w}$ derived from the linear rheology $\left(n^{\prime}=1\right)$ is identical to that derived from any non-linear rheology $\left(n^{\prime}>1\right)$ with (5). It therefore suffices to

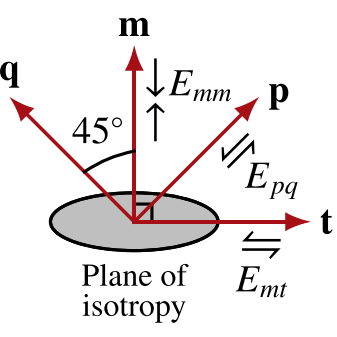

Fig. 2. Axisymmetric polycrystal with longitudinal $\left(E_{m m}\right)$, shear $\left(E_{m t}\right)$ and $45^{\circ}$-shear $\left(E_{p q}\right)$ bulk enhancement factors w.r.t. the symmetry axis $\mathbf{m}$. The transverse direction, $\mathbf{t}$, lies in the plane of isotropy $(\mathbf{t} \perp \mathbf{m})$, while $\mathbf{p}$ is oriented at $45^{\circ}$ to $\mathbf{m}$ and $\mathbf{p} \perp \mathbf{q}$. compare the bulk enhancements resulting from the linear $\left(n^{\prime}=1\right)$ and non-linear $\left(n^{\prime}=3\right)$ realizations of the rheology (1)-(2).

For the sake of simplicity, we restrict ourselves to consider axisymmetric fabrics, which includes single-maximum and girdle fabrics, and denote the fabric symmetry axis $\mathbf{m}$ (Fig. 2). Complementary to $\mathbf{m}$, let $\mathbf{t}$ denote any transverse direction to $\mathbf{m}$, and let $\mathbf{p}$ and $\mathbf{q}$ denote the $\pm 45^{\circ}$ directions to $\mathbf{m}$ : $\mathbf{p}=(\mathbf{m}+\mathbf{t}) / \sqrt{2}$ and $\mathbf{q}=(\mathbf{m}-\mathbf{t}) / \sqrt{2}$. Mechanical deformation tests have found that shearing parallel to the basal planes is about ten times easier for a polycrystal with aligned c-axes compared to isotropic ice (Pimienta and others, 1987), and that shearing of polycrystals with a preferred-direction fabric can be up to $10^{3}-10^{4}$ times softer along the plane with normal $\mathbf{m}$ (i.e. $\mathbf{m}-\mathbf{t}$ shear) compared to the hardest direction, oriented at $\simeq 45^{\circ}$ to $\mathbf{m}$ (i.e. $\mathbf{p}-\mathbf{q}$ shear) (Shoji and Langway, 1985, 1988). Translated into enhancement factors, the two observations suggest that $E_{m t} \simeq 10$ and $E_{m t} / E_{p q} \simeq 10^{3}-10^{4}$. We shall therefore be interested in determining $E_{m t}$ and $E_{p q}$ for axisymmetric polycrystals, and, in addition, the compression/extensional enhancement factor $E_{m m}$. By definition, $E_{m m}$ and $E_{m t}$ are the bulk enhancements of a polycrystal when subject to bulk pure- and simple-shear stresses w.r.t. $\mathbf{m}$ and $\mathbf{t}$ :

$$
E_{m m}=E_{m m}\left(\tau_{0}[\mathbf{I} / 3-\mathbf{m m}]\right)
$$

$$
E_{m t}=E_{m t}\left(\tau_{0}[\mathbf{m t}+\mathbf{t m}]\right),
$$

where $\tau_{0}$ is an arbitrary stress magnitude. Likewise,

$$
E_{p q}=E_{p q}\left(\tau_{0}[\mathbf{p q}+\mathbf{q p}]\right)
$$

A summary of the enhancement factors and defined directions is provided in Figure 2.

At this stage, an important caveat regarding the bulk strain rate (9) is warranted: as pointed out by Meyssonnier and Philip (1996), the mechanical environment of grains in a polycrystal is very different from that of isolated grains (monocrystals) for which (1)-(2) is assumed. Adopting values of $E_{c c}^{\prime}$ and $E_{c a}^{\prime}$ determined from mechanical tests on monocrystals can not necessarily be expected to produce the observed bulk enhancement factors; grain interactions can result in non-preferred slip systems being activated in proportions that are inconsistent with the values $E_{c c}^{\prime}$ and $E_{c a}^{\prime}$ derived from monocrystal mechanical tests. We shall therefore suppose that $E_{c c}^{\prime}$ and $E_{c a}^{\prime}$ are unknown. For a fair comparison between the linear and non-linear rheology (and hence (5) and (2)), $E_{c c}^{\prime}$ and $E_{c a}^{\prime}$ must be selected for both rheologies such that $E_{m t}$ and $E_{m t} / E_{p q}$ match observations in the end-member case of a maximally strong fabric (aligned c-axes). Only after tuning against this end-member case can the bulk directional enhancement factors that result from linear and non-linear grain rheologies be compared for intermediate fabric strengths and the approximation (5) assessed. 



Fig. 3. Bulk enhancement factors $E_{m t}$ (blue-filled contours), $E_{m m}$ (dashed contours) and $E_{m t} / E_{p q}$ (solid contours) for a unidirectional orientation fabric, $\mathbf{c}=\mathbf{m}$, as a function of the grain enhancement factors $E_{c c}^{\prime}$ and $E_{c a}^{\prime}$ in the case of the (a) linear and (b) non-linear grain rheology. Crosses indicate the grain parameters used to model the bulk enhancement factors of an evolving fabric (Fig. 4).

\section{Unidirectional fabric}

In a maximally strong fabric, $\mathrm{c}$-axes are aligned with the symmetry axis, $\mathbf{c}=\mathbf{m}$, and hence $n(\theta, \phi)=\delta(\hat{\mathbf{r}}-\mathbf{m})$, where $\delta(\hat{\mathbf{r}})$ is the Dirac delta function and $\hat{\mathbf{r}}=[\sin (\theta) \cos (\phi), \sin (\theta) \sin (\phi), \cos (\theta)]$ is the radial unit vector. For this distribution, the numerator in (11) is easily determined from (9)-(10) by noting that $\left\langle\mathbf{c}^{k}\right\rangle_{\delta(\hat{\mathbf{r}}-\mathbf{m})}=\mathbf{m}^{k}$. Calculating the denominator in (11) is more tedious but nonetheless possible by rewriting $\mathbf{c}^{k}$ in terms of spherical harmonics, $Y_{l}^{m}(\theta, \phi)$, and using the contraction rule (writing the product of any two spherical harmonics in terms of a new spherical harmonics series). In order to stay focused on the physics, we refer the reader to Supplementary B for details.

In Figure 3, $E_{m t}$ (blue-filled contours), $E_{m t} / E_{p q}$ (solid contours) and $E_{m m}$ (dashed contours) are shown as a function of $E_{c c}^{\prime}$ and $E_{c a}^{\prime}$ in the case of the linear (Fig. 3a) and non-linear (Fig. 3b) grain rheology. With increasing $E_{c a}^{\prime}$ and decreasing $E_{c c}^{\prime}$, the soft-to-hard shear-enhancement ratio, $E_{m t} / E_{p q}$, is generally found to increase, $E_{m m}$ is found to decrease, while $E_{m t}$ monotonically approaches a maximum value (dark blue). Overall, the orientation-dependent fluidity $\left(n^{\prime}=3\right)$, compared to the orientation-independent case $\left(n^{\prime}=1\right.$, or assuming (5)), yields stronger enhancement factors (increased sensitivity to fabric strength). While it is possible to carefully select $E_{c c}^{\prime}$ and $E_{c a}^{\prime}$ for both $n^{\prime}=1$ and $n^{\prime}=3$ such that $E_{m t} / E_{p q} \simeq 10^{3}-10^{4}$ according to Shoji and Langway $(1985,1988)$, the value of $E_{m t}$ is limited to $E_{m t} \leq 2.5$ for $n^{\prime}=1$ and $E_{m t} \leq$ 4.375 for $n^{\prime}=3$, the latter value being approximately a factor of 2 smaller than experimentally determined values (Pimienta and others, 1987).

\section{Evolving single-maximum and girdle fabrics}

Putting aside the upper bounds on $E_{m t}$ (treated in the discussion), the question remains whether (5) can provide a good approximation to fluidity (2) (in the grain-averaged rheology) if they are both tuned to reproduce $E_{m t} / E_{p q} \simeq 10^{3}-10^{4}$ for a unidirectional fabric. For this reason, let us consider a single-maximum and girdle fabric which evolve from isotropy, a situation that is common in polar ice masses.

During vertical pure shear, grains in a polycrystal (ice parcel) tend to rotate towards the compressive axis $(\hat{\mathbf{z}})$ and away from the extensional axis (Azuma and Higashi, 1985; van der Veen and Whillans, 1994), the former coinciding with the symmetry axis, m. The preferred direction (single maximum) therefore strengthens with increasing parcel strain (decreasing ice-parcel height) in the absence of other recrystallization processes (Alley, 1992; Thorsteinsson, 2002). This corresponds to the situation found at ice-sheet domes (Haefeli, 1963; Nye, 1963; Alley and others, 1995) where the vertical strain-rate, $t_{e}^{-1}$, is taken to be approximately uniform, i.e. $\nabla \mathbf{u}=t_{e}^{-1} \operatorname{diag}(1 / 2,1 / 2,-1)$. For girdle fabrics, $\mathrm{c}$-axes are distributed along the preferred plane with normal $\mathbf{m}$, which is the result of extension along $\mathbf{m}$ and compression in the transverse (preferred) plane in the absence of other recrystallization processes (Alley, 1992). The corresponding velocity gradient is the time-reversed of what forms a preferred-direction fabric, i.e. $t_{e} \rightarrow-t_{e}$. The girdle therefore strengthens with increasing ice-parcel height.

In the absence of other microstructural processes, grain rotation can be modelled as a (conservative) convection process on $S^{2}$ involving $n(\theta, \phi)$ (Gödert and Hutter, 1998):

$$
\dot{n}(\theta, \phi)=-\nabla \cdot(n(\theta, \phi) \dot{\mathbf{c}}(\theta, \phi)),
$$

where $\dot{\mathbf{c}}(\theta, \phi)$ is the c-axis velocity field on $S^{2}$, and the divergence operator acts on $S^{2}$. Let us consider the simplest case where grains rotate in response to the macroscopic stretching, $\dot{\boldsymbol{\epsilon}}=\left(\nabla \mathbf{u}+(\nabla \mathbf{u})^{\top}\right) / 2$, and spin, $\boldsymbol{\omega}=\left(\nabla \mathbf{u}-(\nabla \mathbf{u})^{\top}\right) / 2$, which allows the detailed microscopic stress and strain-rate fields to be neglected and hence interactions between neighbouring grains to be disregarded. By moreover neglecting higher-order dependencies on the velocity gradient, and requiring that basal planes preserve their orientation when subject to simple shear (like a deck of cards), it has previously been proposed that (Svendsen and Hutter, 1996; Gödert and Hutter, 1998)

$$
\dot{\mathbf{c}}(\theta, \phi)=\boldsymbol{\omega} \cdot \mathbf{c}-(\dot{\boldsymbol{\epsilon}} \cdot \mathbf{c}-\mathbf{c c} \cdot \dot{\boldsymbol{\epsilon}} \cdot \mathbf{c}),
$$

for an arbitrary c-axis $\mathbf{c}=\mathbf{c}(\theta, \phi)=[\sin (\theta) \cos (\phi), \sin (\theta) \sin (\phi)$, $\cos (\theta)]$.

Notice that (15)-(16) represents a separate kinematic deformation experiment which, given $\nabla \mathbf{u}$, provides an idealized timeevolution of $n(\theta, \phi)$ (and hence $\left\langle\mathbf{c}^{k}\right\rangle$ ) as input for $\left\langle\dot{\boldsymbol{\epsilon}}^{\prime}(\boldsymbol{\tau})\right\rangle$; that is, $\dot{n}(\theta, \phi)$ does not depend on the grain rheology. When dynamically modelling ice flow, the two should be coupled, but for the present purpose it suffices to consider the bulk enhancement factors resulting from a given orientation fabric distribution, $n(\theta, \phi)$.

In order to determine $E_{v w}$ for any fabric, the distribution $n(\theta, \phi)$ must be able to represent structure that is fine enough to account for all the rheology-required structure tensors. For this reason, we expand $n(\theta, \phi)$ in terms of a spherical harmonic series

$$
n(\theta, \phi)=\sum_{l=0}^{L} \sum_{m=-l}^{l} n_{l}^{m} Y_{l}^{m}(\theta, \phi),
$$



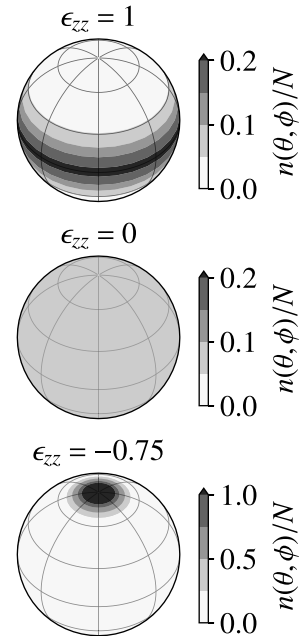

(a)

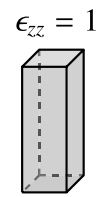

(b)



(c)

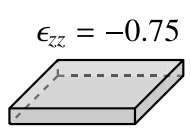

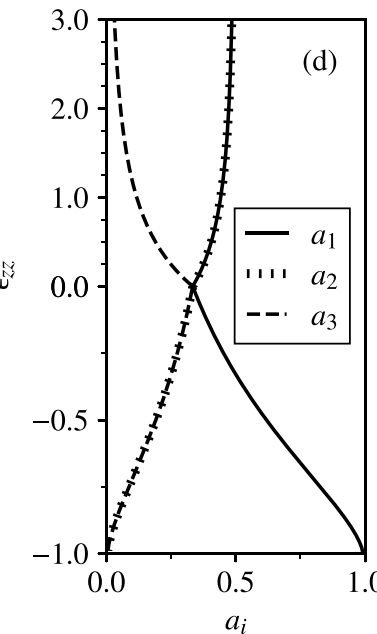

(d)

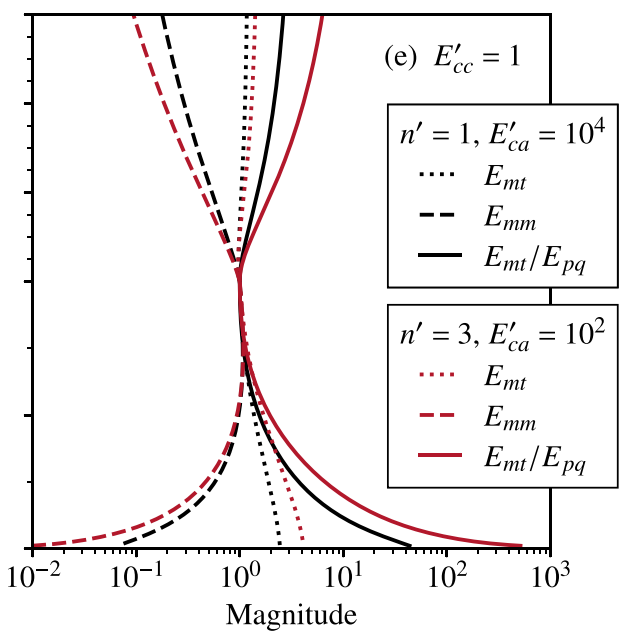

Fig. 4. Vertical pure shear experiment. (a)-(c) Parcel geometries and orientation distributions $n(\theta, \phi)$ at vertical parcel strains of $\epsilon_{z z}=1, \epsilon_{z z}=0$ and $\epsilon_{z z}=-0.75$. (d) Modelled fabric eigenvalues $\left(a_{1}, a_{2}, a_{3}\right)$ using the spectral grain rotation model. (e) Bulk directional enhancement factors given the modelled orientation distribution $n(\theta, \phi)$ for special cases of $E_{c c}^{\prime}$ and $E_{c a}^{\prime}$ (see main text).

where $Y_{l}^{m}(\theta, \phi)$ are the spherical harmonic expansion functions, and $n_{l}^{m}$ are time-dependent expansion coefficients. Because $Y_{l}^{m}(\theta, \phi)$ form a complete orthonormal basis on $S^{2}$, any distribution shape is in principal representable insofar as the expansion series is not truncated $(L \rightarrow \infty)$. In this sense, $n(\theta, \phi)$ is nonparametric since no distribution parameters exist constraining the possible range of distribution shapes. While the expansion (17) provides a more general approach for representing $n(\theta, \phi)$ compared to previous representations based on parametric distribution functions or low-order structure tensors (see Gagliardini and others (2009) and the discussion herein), it is first and foremost a mathematically convenient method that allows for higher-order structure tensors to easily be calculated: the entries of $\left\langle\mathbf{c}^{k}\right\rangle$ are linear combinations of $n_{l}^{m}$ for $l \leq k$ (see Supplementary B).

Let us consider an initially isotropic fabric and truncate the expansion at $L=40$ in order to reduce the influence of regularization on the higher-order structure tensors (see Supplementary B). Figure $4 \mathrm{~d}$ shows the modelled fabric eigenvalues $a_{1}, a_{2}$ and $a_{3}$ of $\left\langle\mathbf{c}^{2}\right\rangle$ as a function of the cumulative parcel strain $\epsilon_{z z}=h / h_{0}-1$, where $h$ and $h_{0}$ are the instantaneous and initial parcel heights, respectively. Here, we assume the usual ordering $a_{1} \geq a_{2} \geq a_{3}$, which implies $a_{1}=a_{2}=a_{3}=1 / 3$ for isotropic fabrics, $1>a_{1}>1 / 3$ $>a_{2}=a_{3} \geq 0$ for axisymmetric preferred-direction fabrics, and $1>a_{1}=a_{2}>1 / 3>a_{3} \geq 0$ for axisymmetric preferred-plane (girdle) fabrics (Woodcock, 1977). For reference, Figures 4a to $4 \mathrm{c}$ show the specific parcel geometries and normalized $n(\theta, \phi)$ at $\epsilon_{z z}=1$, $\epsilon_{z z}=0$ and $\epsilon_{z z}=-0.75$, respectively.

In Figure $4 \mathrm{e}$, the corresponding $E_{m t}$ (dotted), $E_{m m}$ (dashed) and $E_{m t} / E_{p q}$ (full) are plotted for $\left(n^{\prime}, E_{c c}^{\prime}, E_{c a}^{\prime}\right)=\left(1,1,10^{4}\right)$ in black lines and $\left(n^{\prime}, E_{c c}^{\prime}, E_{c a}^{\prime}\right)=\left(3,1,10^{2}\right)$ in red lines (crosses in Figs $3 \mathrm{a}, 3 \mathrm{~b}$ ). Choosing $E_{c c}^{\prime}=1$ implies grains are equally hard to compress along $\mathbf{c}$ and $\mathbf{a}$, which is a reasonable assumption (Gillet-Chaulet and others, 2005), and $E_{c a}^{\prime}$ was subsequently picked to reproduce $E_{m t} / E_{p q}=10^{4}$ for a unidirectional fabric (the ideal limit of $\left.\epsilon_{z z}=-1\right)$. For parcel strains of $-0.4<\epsilon_{z z}<0.1$, the linear $\left(n^{\prime}=1\right.$, or assuming (5)) and non-linear $\left(n^{\prime}=3\right)$ solutions are found to agree, while for stronger fabrics (larger absolute strains), the non-linear rheology produces bulk enhancements that scale faster with fabric strength compared to the linear rheology. Although $E_{m t}$ (dotted lines) approaches the upper limits found for a unidirectional fabric as $\epsilon_{z z} \rightarrow-1$, the ratios $E_{m t} / E_{p q}$ (full lines) remain far from $10^{4}$. This discrepancy is the result of $n(\theta, \phi)$ never becoming $\delta(\hat{\boldsymbol{r}}-\mathbf{m})$ due to non-negligible regularization for $\epsilon_{z z}<-0.8$ (see Supplementary B). In the limit $L \rightarrow \infty$, no regularization is required, and we expect that $n(\theta, \phi) \rightarrow \delta(\hat{\boldsymbol{r}}-\mathbf{m}) \quad$ as $\epsilon_{z z} \rightarrow-1$. Nonetheless, for the intermediate-to-strong fabrics that we are able to represent, we find that the bulk enhancement factors produced by a linear grain rheology (and therefore also (5)) scales more slowly with fabric strength compared to $n^{\prime}=3$. Specifically, we find that the non-linear-to-linear ratios of $E_{m t} / E_{p q}$ and $E_{m m}$ can be at least 10 and 0.1 , respectively.

\section{Discussion}

A shortcoming of our study is our one-sided focus on the Sachs (homogeneous stress) assumption, which does not allow for $E_{m t} \simeq 10$ in accordance with Pimienta and others (1987). Although calculating bulk enhancement factors for both the Sachs and Taylor end-member cases would allow for a more complete assessment of the approximation (5), we note that the Sachs assumption is arguably the better of the two simple homogenization schemes (see above). Modelling efforts (Pettit and others, 2007; Martín and others, 2009; Martín and Gudmundsson, 2012; Hruby and others, 2020) that take the Sachs grain-averaged rheology (9) with (5) as the bulk rheology might therefore underor overestimate bulk strain-rate enhancements by at least an order of magnitude for intermediate-to-strong fabrics.

Other studies have adopted the orthotropic flow law proposed by Gillet-Chaulet and others (2005), which relies on the viscoplastic self-consistent (VPSC) homogenization scheme (Meyssonnier and Philip, 1996) to infer the bulk directional enhancement factors of the orthotropic rheology. The VPSC approach represents a compromise between the Sachs and Taylor assumptions and does not necessarily restrict $E_{m t}$, but requires an iterative numerical procedure to determine (11). Insofar as the VPSC approach is based on the grain rheology (1) with (5), such as proposed by Gillet-Chaulet and others (2005) and adopted in several modelling studies (Gillet-Chaulet and others, 2006; Durand and others, 2007; Ma and others, 2010), our above caveats apply, too, although to what extent is less clear. Castelnau and others (1998) showed that the VPSC approach based on a basal slip (Schmid) grain rheology with an orientation-dependent fluidity can reproduce observed strain-rate enhancements derived from mechanical tests on the GRIP ice core (single-maximum fabrics). 
The strongest GRIP fabric occurs at a depth of approximately $2500 \mathrm{~m}$ where $a_{1} \simeq 0.95$. For this fabric, the VPSC approach gave $E_{m m} \simeq 0.03$ and $E_{m t}^{\dagger}=12$, compared to $E_{m m}=0.03$ and $E_{m t}^{\dagger}=65$ determined from mechanical tests, where $E_{m t}^{\dagger}=E_{m t}\left(\tau_{0}[\mathbf{I} / 3-\mathbf{p p}]\right)$ is the $\mathbf{m}-\mathbf{t}$ shear enhancement under uniaxial compression along $\mathbf{p}$. In comparison, for $n^{\prime}=3$ we find $E_{m m}=0.05$ and $E_{m t}^{\dagger}=3$ (latter not shown) given $n(\theta, \phi)$ corresponding to $a_{1} \simeq 0.95$, although the enhancement-factor definition used by Castelnau and others (1998) is different from ours which might partly explain the discrepancy.

Given a unidirectional fabric and the Sachs assumption, Gödert and Hutter (1998) and Gagliardini and Meyssonnier (1999) previously reported $E_{m t} \leq 2.5$ using a linear grain rheology akin to the present. Thorsteinsson (2001) considered the same configuration and reported that a basal slip (Schmid) model produces $E_{m t} \leq 4.375$ for an orientation-dependent fluidity with a power-law exponent of three. For non-unidirectional fabrics, however, we emphasize that the rheology (1)-(2) and a Schmid model will generally not produce the same bulk directional enhancement factors (Thorsteinsson, 2001): in the limit of incompressibility along $\mathbf{c}$ (i.e. $E_{c c}^{\prime}=0$ ), longitudinal straining in the crystal basal plane is still possible with (1)-(2) (Supplementary A), unlike a Schmid model where only basal-plane shear is permitted.

We end with an outlook on the possibility of including our work in large-scale ice-flow models; i.e. by using (9)-(10) as is, or to determine bulk directional enhancement factors for another bulk rheology (e.g. the orthotropic flow law). The main obstacle is that $n(\theta, \phi)$ must be able to represent structure that is fine enough to account for all the rheology-required structure tensors. A popular fabric model is to represent $n(\theta, \phi)$ as a series expansion in terms of structure tensors (Gödert, 2003; Gillet-Chaulet and others, 2005) by noting that any $n(\theta, \phi)$ can be expressed as a linear combination of even-ordered structure tensors (Advani and Tucker, 1987). In such models, calculating the time-evolution of $n(\theta, \phi)$ requires specifying $\mathrm{d}\left\langle\mathbf{c}^{k}\right\rangle / \mathrm{d} t$, but for closure reasons $\left\langle\mathbf{c}^{k}\right\rangle$ for $k \geq 4$ tend to be parameterized in terms of lower-order structure tensors. Unless $\left\langle\mathbf{c}^{k}\right\rangle$ for $k \leq 8$ are allowed to freely evolve (not parameterized), calculated bulk enhancement factors may not agree with ours. Implementing our spectral grain rotation model could therefore provide a useful path forward. To that end, we note that truncating $n(\theta, \phi)$ at e.g. $L=8,16$, or 32 implies solving a 45-, 153- or 561-dimensional linear advection-reactiondiffusion problem per grid point, respectively.

\section{Conclusion}

We investigated how the orientation-dependent non-linear fluidity of a transversely isotropic grain rheology can influence the directional enhancement factors of a polycrystal by assuming a homogeneous stress field over the polycrystal scale (Sachs hypothesis). For polycrystals with intermediate-to-strong orientation fabrics, we find that the soft-to-hard shear-enhancement ratio, $E_{m t} / E_{p q}$, of axisymmetric polycrystals can be at least ten times larger compared to if the fluidity is orientation independent, while the compressional/extensional enhancement along the fabric symmetry direction, $E_{m m}$, can be at least ten times smaller. Care should therefore be exercised in large-scale ice-flow modelling efforts that either (i) identify the (Sachs) grain-averaged transversely isotropic rheology as the bulk rheology, or (ii) rely on it to derive bulk directional enhancement factors for another anisotropic bulk rheology. Our results thus extend previous work on grain-averaged rheologies with orientation-dependent fluidities (Thorsteinsson (2001) and Pettit and others (2007), among others) to the transversely isotropic grain rheology, which has implications for large-scale anisotropic ice-flow modelling. Finally, because the orientation-dependent fluidity causes the grain-averaged rheology to depend on higher-order fabric structure tensors, we proposed a new non-parametric spectral fabric model that allows for higher-order structure tensors to easily be calculated for arbitrary orientation fabrics.

Acknowledgements. We thank two anonymous reviewers for comments that helped improve this manuscript greatly, and Sérgio H. Faria for his valuable comments on the spectral fabric model. The research leading to these results has received funding from the Villum Investigator grant No. 16572 as part of the IceFlow project and the European Research Council under the European Community's Seventh Framework Programme (FP7/2007-2013) / ERC grant agreement 610055 as part of the ice2ice project.

Supplementary material. The supplementary material for this article can be found at https://doi.org/10.1017/jog.2020.117.

\section{References}

Advani SG and Tucker CL (1987) The use of tensors to describe and predict fiber orientation in short fiber composites. Journal of Rheology 31(8), 751784. doi: 10.1122/1.549945.

Alley RB (1992) Flow-law hypotheses for ice-sheet modeling. Journal of Glaciology 38(129), 245-256. doi: 10.3189/S0022143000003658.

Alley RB and 5 others (1995) Comparison of deep ice cores. Nature 373 (6513), 393-393. doi: https://doi.org/10.1038/373393b0.

Azuma N (1994) A flow law for anisotropic ice and its application to ice sheets. Earth and Planetary Science Letters 128(3), 601-614. doi: https:// doi.org/10.1016/0012-821X(94)90173-2.

Azuma N (1995) A flow law for anisotropic polycrystalline ice under uniaxial compressive deformation. Cold Regions Science and Technology 23(2), 137147. doi: https://doi.org/10.1016/0165-232X(94)00011-L.

Azuma N and Higashi A (1985) Formation processes of ice fabric pattern in ice sheets. Annals of Glaciology 6, 130-134. doi: 10.3189/1985AoG61-130-134.

Castelnau O and 7 others (1998) Anisotropic behavior of GRIP ices and flow in Central Greenland. Earth and Planetary Science Letters 154(1), 307-322. doi: https://doi.org/10.1016/S0012-821X(97)00193-3.

Castelnau O, Duval P, Lebensohn RA and Canova GR (1996) Viscoplastic modeling of texture development in polycrystalline ice with a self-consistent approach: Comparison with bound estimates. Journal of Geophysical Research: Solid Earth 101(B6), 13851-13868. doi: https://doi.org/10.1029/96JB00412.

Castelnau O, Duval P, Montagnat M and Brenner R (2008) Elastoviscoplastic micromechanical modeling of the transient creep of ice. Journal of Geophysical Research: Solid Earth 113(B11). doi: https://doi.org/10.1029/ 2008JB005751.

Durand G and 8 others (2007) Change in ice rheology during climate variations - implications for ice flow modelling and dating of the EPICA Dome C core. Climate of the Past 3(1), 155-167. doi: 10.5194/cp-3-155-2007.

Duval P, Ashby MF and Anderman I (1983) Rate-controlling processes in the creep of polycrystalline ice. The Journal of Physical Chemistry 87(21), 40664074. doi: $10.1021 / \mathrm{j} 100244 \mathrm{a} 014$.

Fan S and 7 others (2020) Temperature and strain controls on ice deformation mechanisms: insights from the microstructures of samples deformed to progressively higher strains at $-10,-20$ and $-30^{\circ} \mathrm{C}$. The Cryosphere 14, 3875-3905. doi: 10.5194/tc-14-3875-2020.

Faria SH, Weikusat I and Azuma N (2014) The microstructure of polar ice. Part II: state of the art. Journal of Structural Geology 61, 21-49. doi: https:// doi.org/10.1016/j.jsg.2013.11.003.

Gagliardini O, Gillel-Chaulet F and Montagnat M (2009) A review of anisotropic polar ice models: from crystal to ice-sheet flow models. Low Temperature Science 68(suppl., 149-166.

Gagliardini O and Meyssonnier J (1999) Analytical derivations for the behavior and fabric evolution of a linear orthotropic ice polycrystal. Journal of Geophysical Research: Solid Earth 104(B8), 17797-17809. doi: https://doi. org/10.1029/1999JB900146.

Gillet-Chaulet F, Gagliardini O, Meyssonnier J, Montagnat M and Castelnau O (2005) A user-friendly anisotropic flow law for ice-sheet modeling. Journal of Glaciology 51(172), 3-14. doi: 10.3189/172756505781829584.

Gillet-Chaulet F, Gagliardini $O$, Meyssonnier $J$, Zwinger $T$ and Ruokolainen J (2006) Flow-induced anisotropy in polar ice and related icesheet flow modelling. Journal of Non-Newtonian Fluid Mechanics 134(1), 33-43. doi: https://doi.org/10.1016/j.jnnfm.2005.11.005. 
Gödert G (2003) A mesoscopic approach for modelling texture evolution of polar ice including recrystallization phenomena. Annals of Glaciology 37, 23-28. doi: 10.3189/172756403781815375.

Gödert G and Hutter K (1998) Induced anisotropy in large ice shields: theory and its homogenization. Continuum Mechanics and Thermodynamics 10 (5), 293-318. doi: https://doi.org/10.1007/s001610050095.

Goldsby D and Kohlstedt D (1997) Grain boundary sliding in fine-grained Ice I. Scripta Materialia 37(9), 1399-1406. doi: https://doi.org/10.1016/ S1359-6462(97)00246-7.

Haefeli R (1963) A numerical and experimental method for determining ice motion in the central parts of ice sheets. International Association of Scientific Hydrology Publication 61 (General Assembly of Berkeley 1963Snow and Ice), pp. 253-260.

Hruby $\mathbf{K}$ and 5 others (2020) The impact of temperature and crystal orientation fabric on the dynamics of mountain glaciers and ice streams. Journal of Glaciology 66(259), 755-765. doi: 10.1017/jog.2020.44.

Johnson A (1977) Creep characterization of transversely-isotropic metallic materials. Journal of the Mechanics and Physics of Solids 25(2), 117-126. doi: https://doi.org/10.1016/0022-5096(77)90007-2.

Kamb WB (1961) The glide direction in ice. Journal of Glaciology 3(30), 1097 1106. doi: $10.3189 /$ S0022143000017500.

Kuiper EJN and 5 others (2020a) Using a composite flow law to model deformation in the NEEM deep ice core, Greenland: Part 1 the role of grain size and grain size distribution on the deformation of Holocene and glacial ice. The Cryosphere 14, 2429-2448. doi: 10.5194/tc-14-2429-2020.

Kuiper EJN and 5 others (2020b) Using a composite flow law to model deformation in the NEEM deep ice core, Greenland: Part 2 the role of grain size and premelting on ice deformation at high homologous temperature. The Cryosphere 14, 2449-2467. doi: 10.5194/tc-14-2449-2020.

Ma Y and 5 others (2010) Enhancement factors for grounded ice and ice shelves inferred from an anisotropic ice-flow model. Journal of Glaciology 56(199), 805-812. doi: 10.3189/002214310794457209.

Martín C and Gudmundsson GH (2012) Effects of nonlinear rheology, temperature and anisotropy on the relationship between age and depth at ice divides. The Cryosphere 6(5), 1221-1229. doi: 10.5194/tc-61221-2012.

Martín C, Gudmundsson GH, Pritchard HD and Gagliardini O (2009) On the effects of anisotropic rheology on ice flow, internal structure, and the age-depth relationship at ice divides. Journal of Geophysical Research: Earth Surface 114(F4). doi: https://doi.org/10.1029/2008JF001204.

Meyssonnier J and Philip A (1996) A model for the tangent viscous behaviour of anisotropic polar ice. Annals of Glaciology 23, 253-261. doi: 10.3189/ S0260305500013513.

Montagnat M and 11 others (2014) Multiscale modeling of ice deformation behavior. Journal of Structural Geology 61, 78-108. doi: https://doi.org/10. 1016/j.jsg.2013.05.002.
Nye JF (1963) Correction factor for accumulation measured by the thickness of the annual layers in an ice sheet. Journal of Glaciology 4(36), 785-788. doi: $10.3189 /$ S0022143000028367.

Pettit EC, Thorsteinsson T, Jacobson HP and Waddington ED (2007) The role of crystal fabric in flow near an ice divide. Journal of Glaciology 53 (181), 277-288. doi: 10.3189/172756507782202766.

Pimienta P, Duval P and Lipenkov VY (1987) Mechanical behaviour of anisotropic polar ice. In Waddington ED and Walder JS eds. The Physical Basis of Ice Sheet Modelling, IAHS Publication No. 170, Wallingford, UK: IAHS Press, pp. 57-66.

Sachs G (1929) Zur Ableitung einer Fliessbedingung. Berlin, Heidelberg: Springer, pp. 94-97. doi: 10.1007/978-3-642-92045-5_12.

Shoji H and Langway CC (1985) Mechanical Properties of Fresh Ice Core from Dye 3, Greenland. American Geophysical Union (AGU), pp. 39-48. doi: https://doi.org/10.1029/GM033p0039.

Shoji H and Langway CC (1988) Flow-law parameters of the dye 3, Greenland, deep ice core. Annals of Glaciology 10, 146-150. doi: 10.3189/ S026030550000433X.

Suquet P and 7 others (2012) Multi-scale modeling of the mechanical behavior of polycrystalline ice under transient creep. Procedia International Union of Theoretical and Applied Mechanics 3, 76-90. doi: https://doi.org/ 10.1016/j.piutam.2012.03.006, iUTAM Symposium on Linking Scales in Computations: From Microstructure to Macro-scale Properties.

Svendsen B and Hutter K (1996) A continuum approach for modelling induced anisotropy in glaciers and ice sheets. Annals of Glaciology 23, 262-269. doi: 10.3189/S0260305500013525.

Taylor GI (1938) Plastic strain in metals. Journal of the Institute of Metals 62, 307-324.

Thorsteinsson T (2001) An analytical approach to deformation of anisotropic ice-crystal aggregates. Journal of Glaciology 47(158), 507-516. doi: 10.3189/ 172756501781832124.

Thorsteinsson T (2002) Fabric development with nearest-neighbor interaction and dynamic recrystallization. Journal of Geophysical Research: Solid Earth 107(B1), ECV 3-1-ECV 3-13. doi: https://doi.org/10.1029/ 2001JB000244

Thorsteinsson T, Waddington ED and Fletcher RC (2003) Spatial and temporal scales of anisotropic effects in ice-sheet flow. Annals of Glaciology 37, 40-48. doi: 10.3189/172756403781815429.

van der Veen C and Whillans I (1994) Development of fabric in ice. Cold Regions Science and Technology 22(2), 171-195. doi: https://doi.org/10. 1016/0165-232X(94)90027-2.

Weertman J (1973) Creep of ice. In Whalley E Jones SJ and Gold LW eds. Physics and Chemistry of Ice. Ottawa, Canada: Royal Society of Canada, pp. 320-337.

Woodcock NH (1977) Specification of fabric shapes using an eigenvalue method. Geological Society of America Bulletin 88(9), 1231-1236. doi: 10. 1130/0016-7606(1977)88;1231:SOFSUA;2.0.CO;2. 